Supporting Information for:

\title{
Leveraging Stereoelectronic Effects in Biofilm Eradication: Synthetic $\beta$-Amino Human Milk Oligosaccharides Impede Microbial Adhesion as Observed by Scanning Electron Microscopy
}

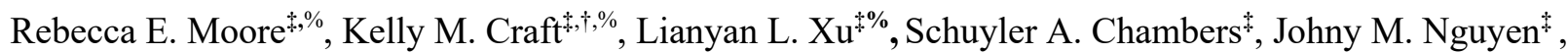
Keevan C. Marion", Jennifer A. Gaddy ${ }^{\S}, \|^{*}$, and Steven D. Townsend ${ }^{\ddagger, *}$

‡Department of Chemistry, Vanderbilt University, 7330 Stevenson Center, Nashville, Tennessee 37235, United States

Email: steven.d.townsend@vanderbilt.edu

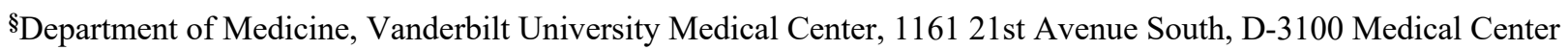
North, Nashville, Tennessee 37232, United States

Email: jennifer.a.gaddy@vumc.org

"Tennessee Valley Healthcare Systems, Department of Veterans Affairs, 1310 24th Avenue South, Nashville,

Tennessee 37212, United States

\section{Table of Contents}

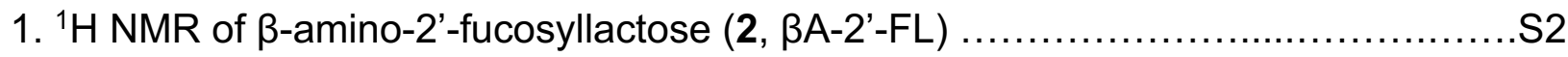

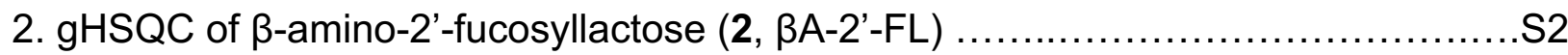

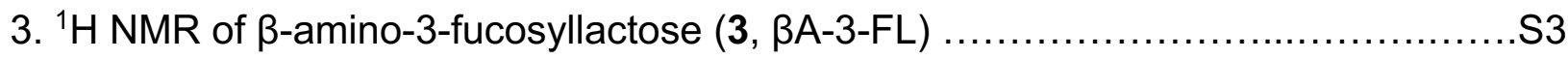

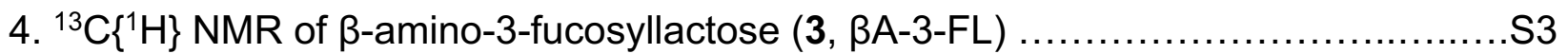

5. gHSQC of $\beta$-amino-3-fucosyllactose $(3, \beta A-3-F L) ~ \ldots \ldots \ldots \ldots \ldots \ldots \ldots \ldots \ldots \ldots \ldots . . . \ldots \ldots$

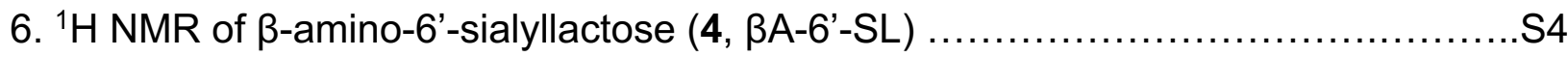

7. ${ }^{13} \mathrm{C}\left\{{ }^{1} \mathrm{H}\right\}$ NMR of $\beta$-amino-6'-sialyllactose $(4, \beta A-6$ '-SL) .............................S5

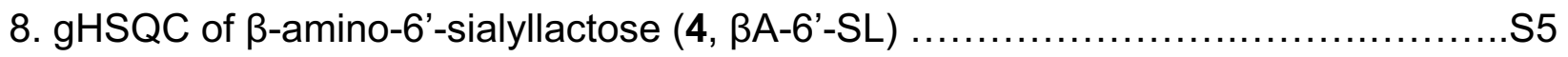

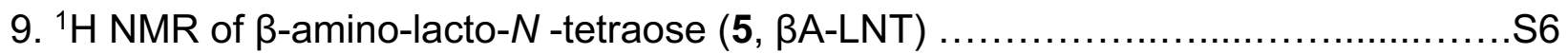

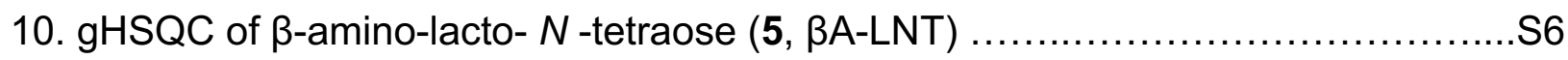

11. Table S1. Anomeric Proton Shifts to determine HMO: $\beta A-H M O$ Ratio ................S7 
${ }^{1} \mathrm{H}$ NMR $(600 \mathrm{MHz}, \mathrm{MeOD})$ of 2

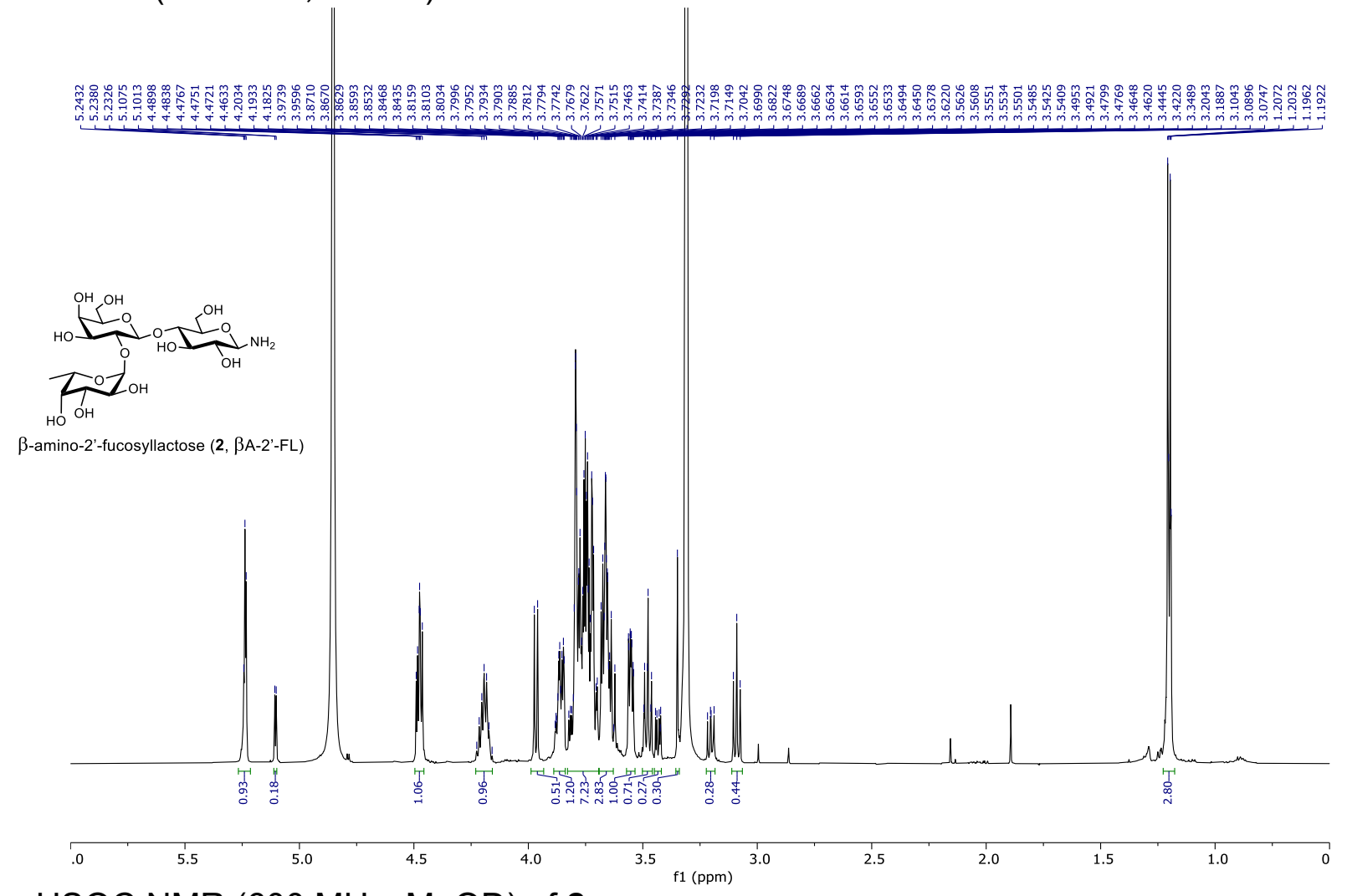

gHSQC NMR (600 MHz, MeOD) of 2

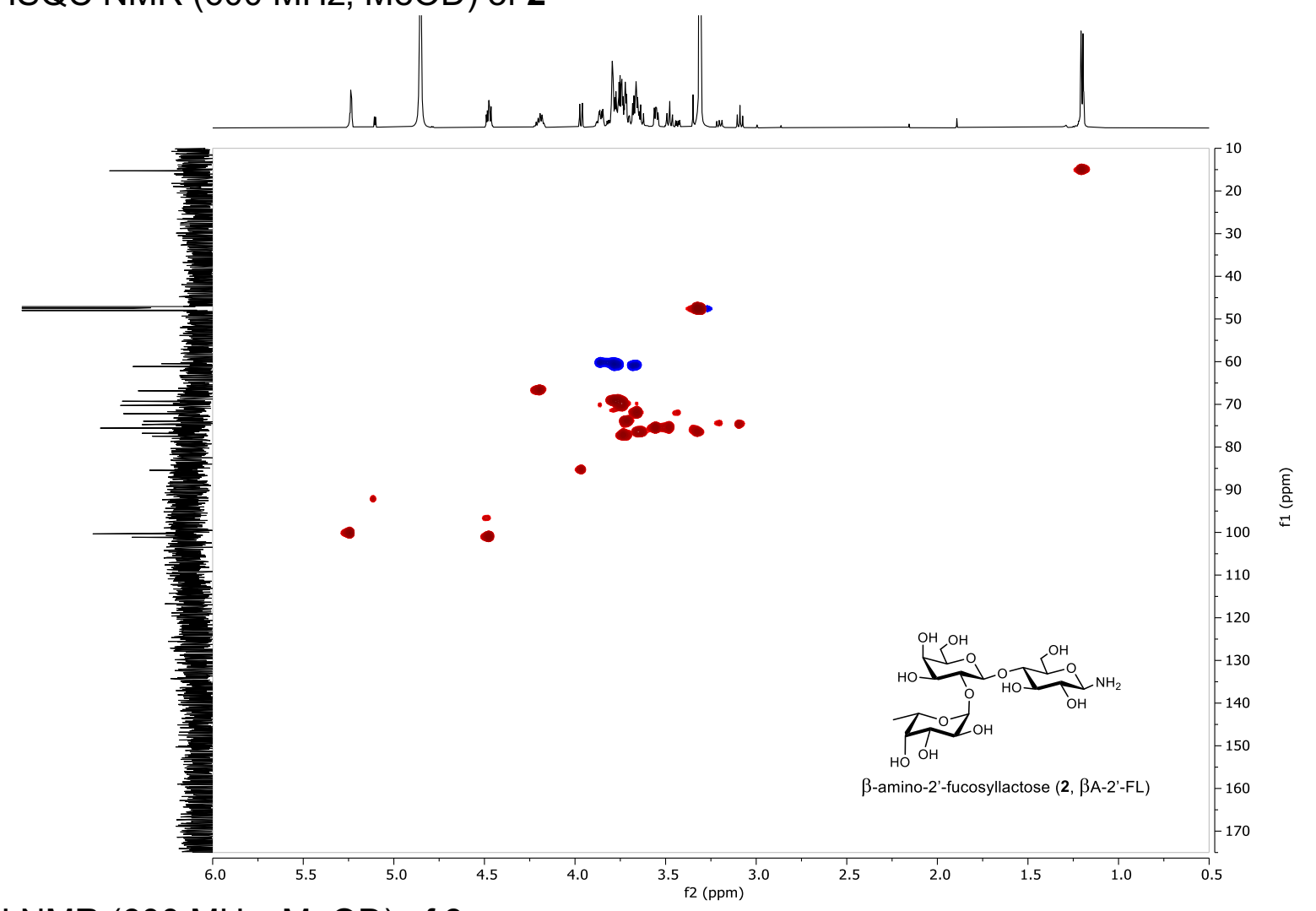

${ }^{1} \mathrm{H}$ NMR (600 MHz, MeOD) of 3 


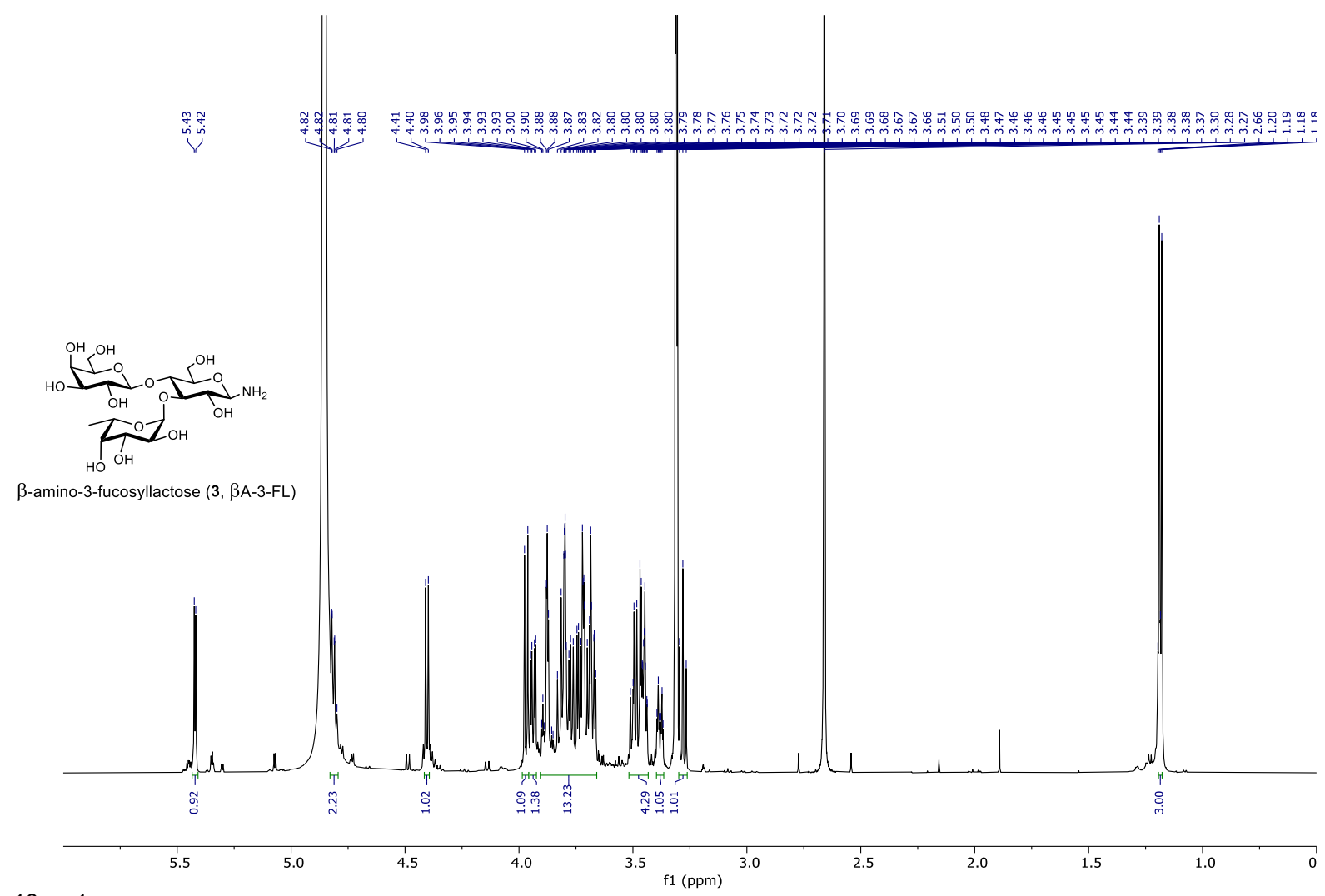

${ }^{13} \mathrm{C}\left\{{ }^{1} \mathrm{H}\right\}$ NMR (151 MHz, MeOD) of 3
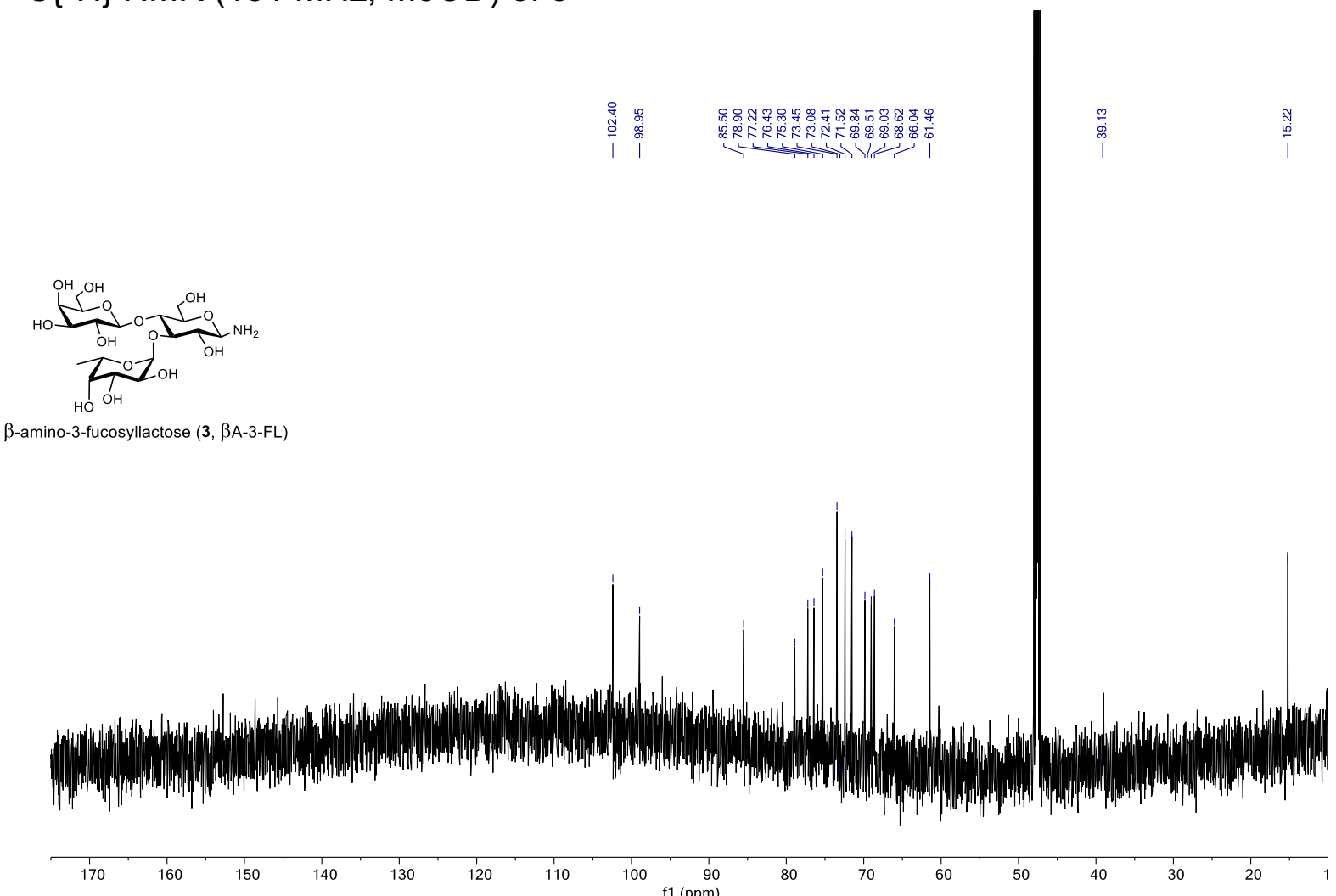

gHSQC NMR (600 MHz, MeOD) of 3 


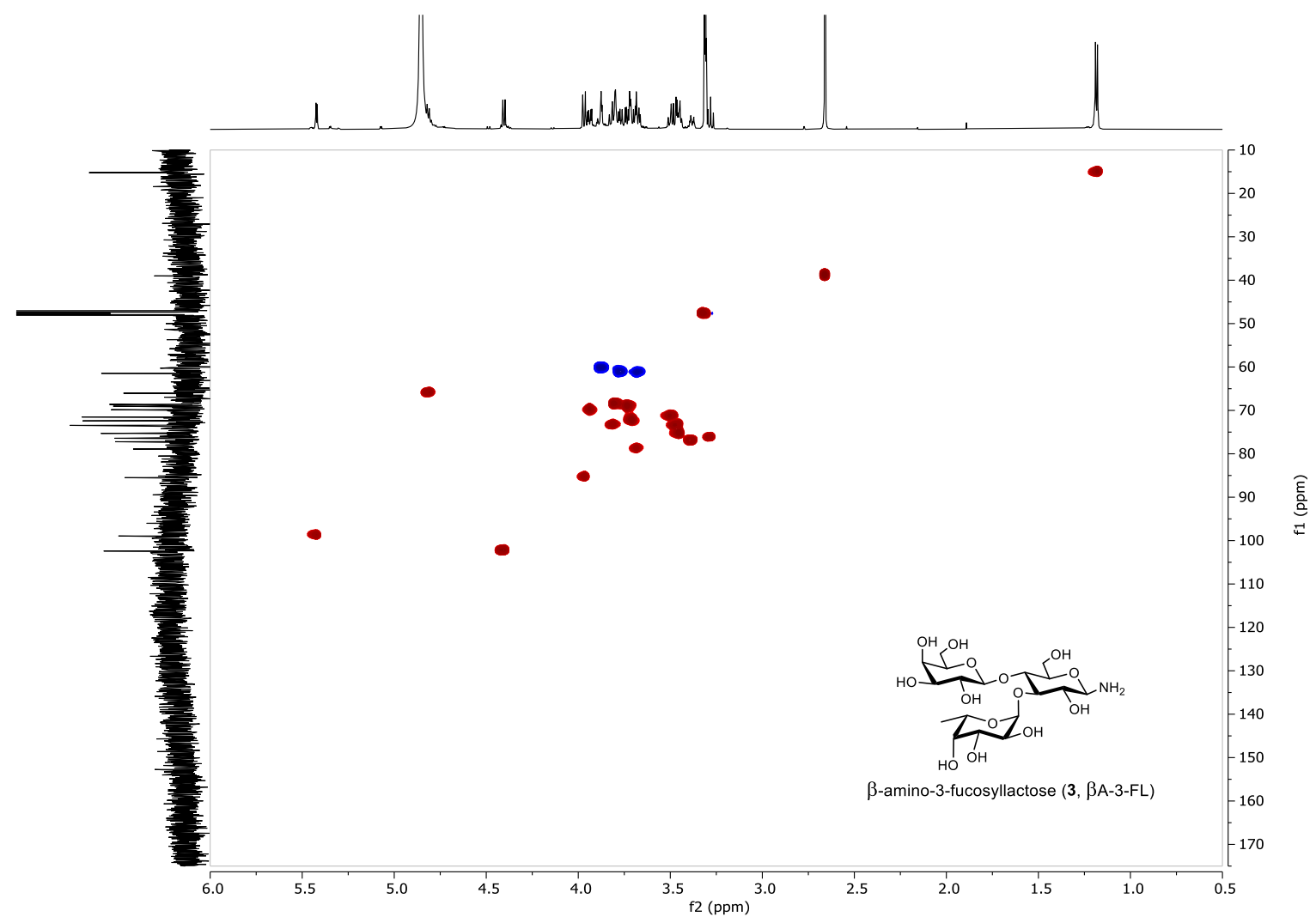

${ }^{1} \mathrm{H}$ NMR (600 MHz, MeOD) of 4

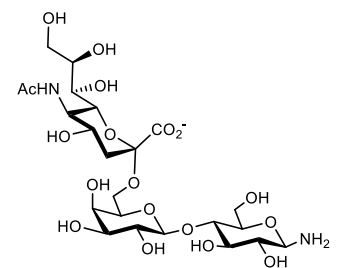

$\beta$-amino-6'-sialyllactose $(4, \beta A-6$ '-SL)

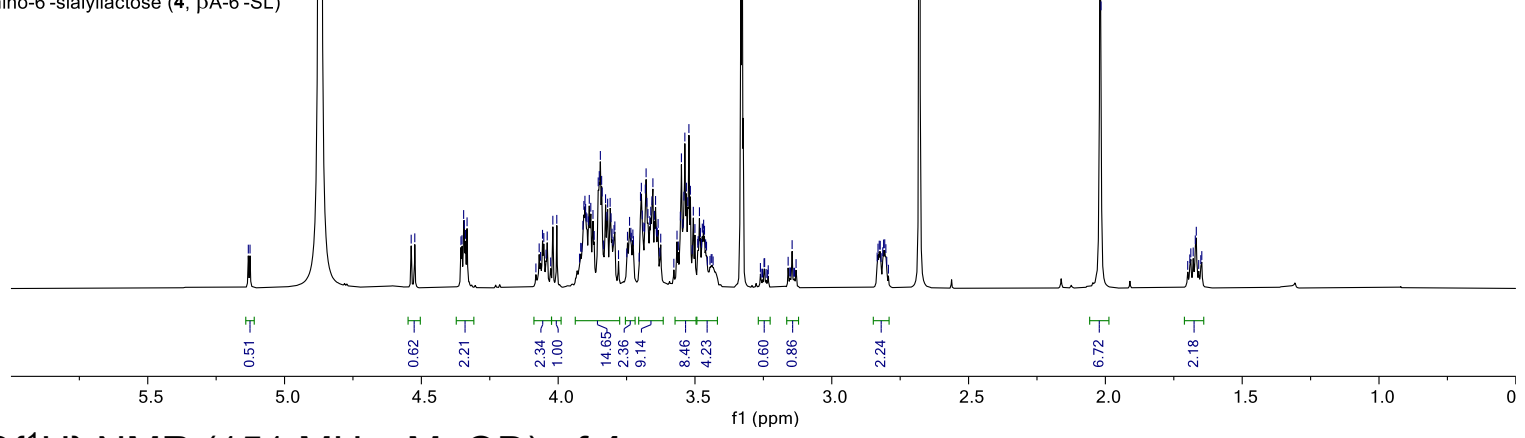

${ }^{13} \mathrm{C}\left\{{ }^{1} \mathrm{H}\right\}$ NMR (151 MHz, MeOD) of 4

S4 


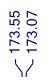

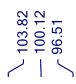

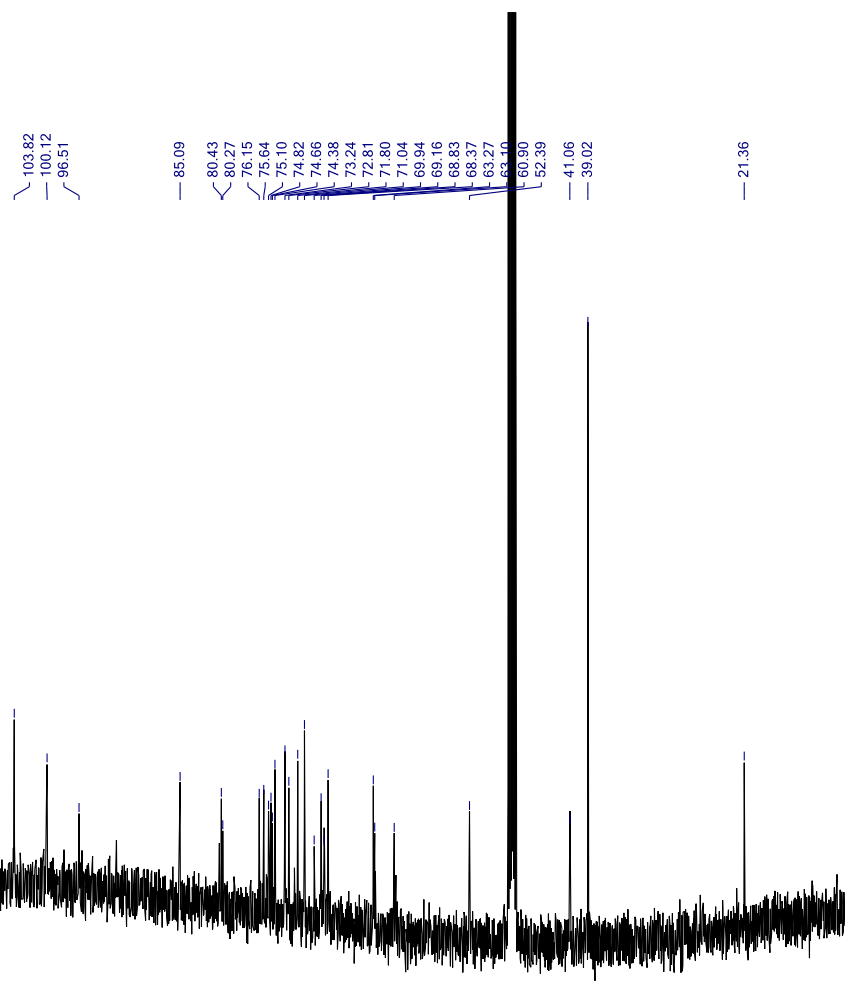

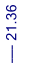

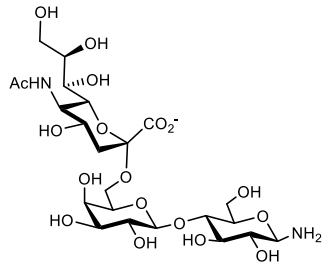

$\beta$-amino-6'-sialyllactose (4, $\beta \mathrm{A}-6^{\prime}$-SL)

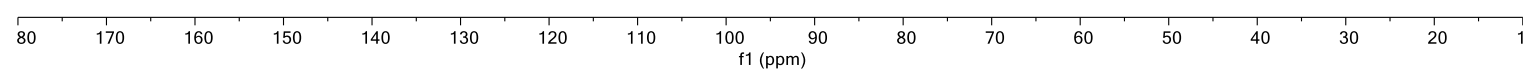

gHSQC NMR (600 MHz, MeOD) of 4

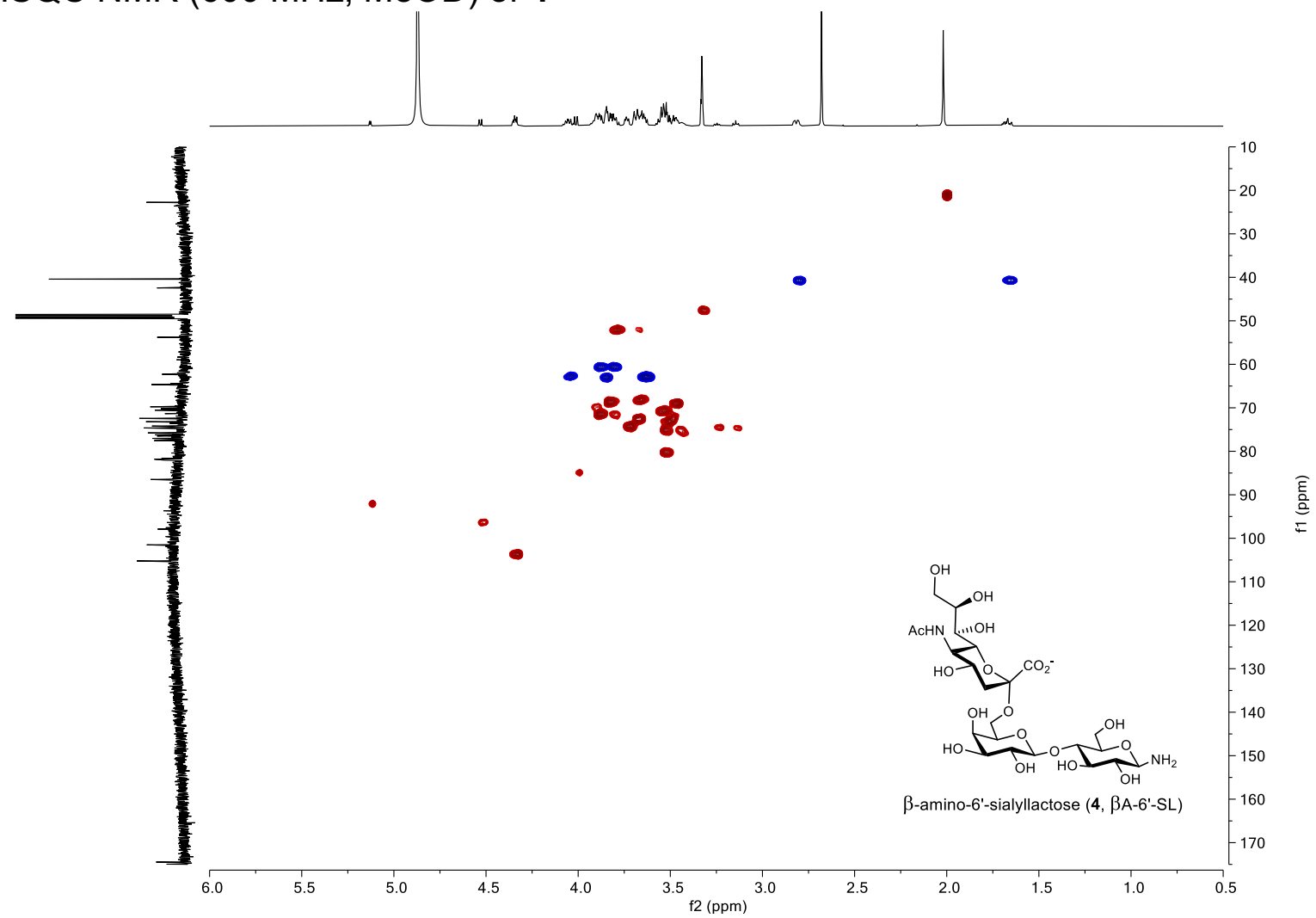

${ }^{1} \mathrm{H}$ NMR (600 MHz, MeOD) of 5

S5 

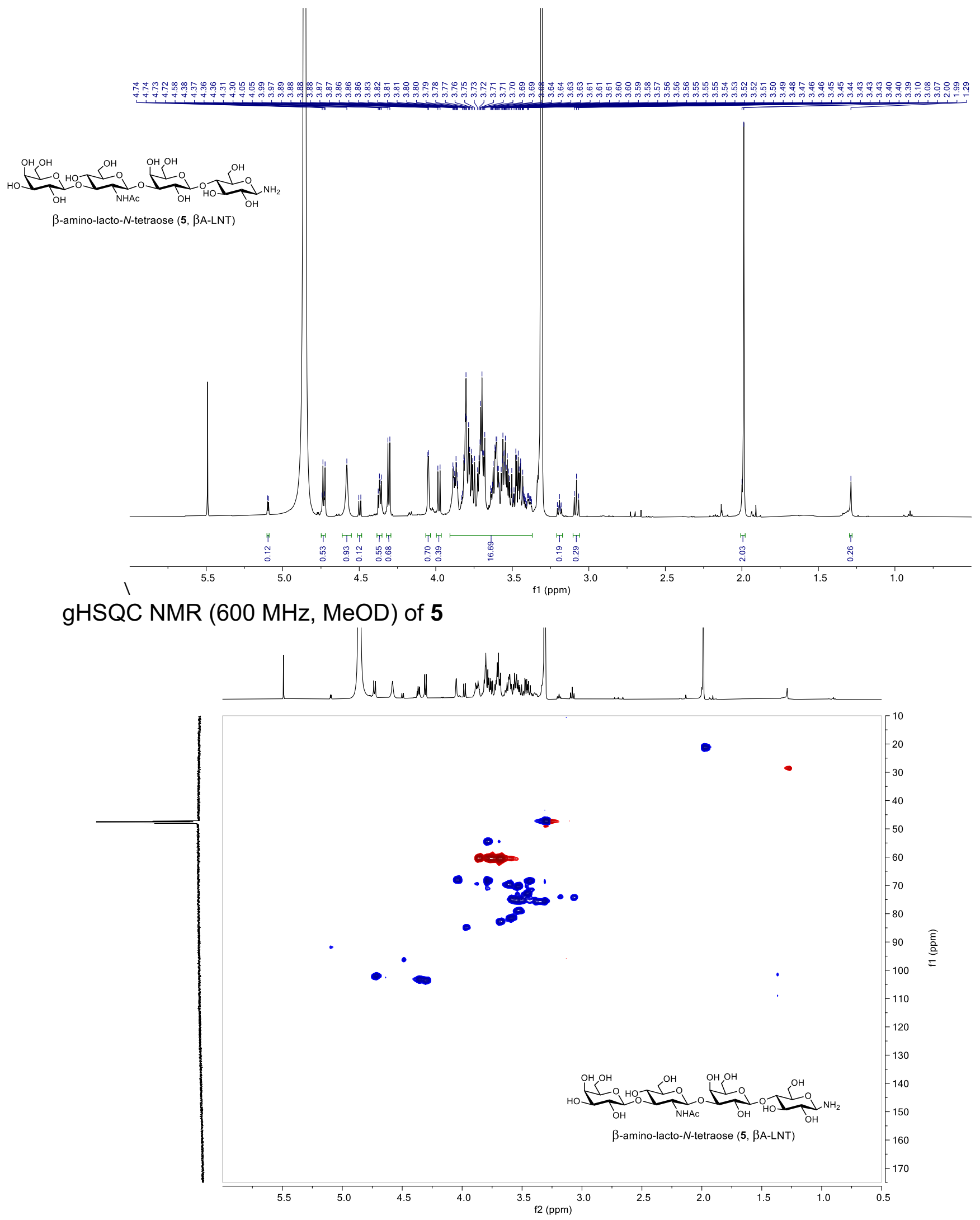

S6 
Table S1. Anomeric Proton Shifts to determine HMO: $\beta A-H M O$ Ratio based on ${ }^{1} \mathrm{H}$ NMR

\begin{tabular}{|c|c|c|c|}
\hline$\underline{H M O}$ & HMO shift (ppm) & $\beta A-\mathrm{HMO}$ & $\begin{array}{c}\beta A-\mathrm{HMO} \text { shift } \\
(\mathrm{ppm})\end{array}$ \\
\hline 2' $-\mathrm{FL}^{\prime}$ & 5.10 & $\beta A-2^{\prime}-\mathrm{FL}$ & 3.97 \\
\hline $3-\mathrm{FL}$ & 5.07 & $\beta A-3-\mathrm{FL}$ & 3.97 \\
\hline 6' $-\mathrm{SL}$ & 5.13 & $\beta A-6^{\prime}-\mathrm{SL}$ & 4.01 \\
\hline LNT & 5.10 & $\beta A-\mathrm{LNT}$ & 3.98 \\
\hline
\end{tabular}

${ }^{*}{ }^{1} \mathrm{H}$ NMR data obtained in MeOD 